\title{
A Function of Time, Frequency, Lag, and Doppler
}

\author{
Jeffrey C. O'Neill, Member, IEEE, and William J. Williams, Senior Member, IEEE
}

\begin{abstract}
In signal processing, four functions of one variable are commonly used. They are the signal in time, the spectrum, the auto-correlation function of the signal, and the auto-correlation function of the spectrum. The variables of these functions are denoted, respectively, as time, frequency, lag, and doppler. In time-frequency analysis, these functions of one variable are extended to quadratic functions of two variables. In this paper, we investigate a method for creating quartic functions of three of these variables as well as a quartic function of all four variables. These quartic functions provide a meaningful representation of the signal that goes beyond the well-known quadratic functions. The quartic functions are applied to the design of signal-adaptive kernels for the Cohen class and shown to provide improvements over previous methods.
\end{abstract}

\section{INTRODUCTION}

A SIGNAL $x(t)$ and its spectrum $X(\omega)$ are two commonly used linear representations of a signal. The spectrum is obtained by applying a Fourier transform to the signal

$$
X(\omega)=\int x(t) e^{-j \omega t} d t .
$$

Two commonly used quadratic functions of the signal are the auto-correlation of the signal and the auto-correlation of the spectrum

$$
\begin{aligned}
r_{x}(\tau) & =\int x(t) x^{*}(t+\tau) d t \\
r_{X}(\theta) & =\int X(\omega) X^{*}(\omega+\theta) d \omega .
\end{aligned}
$$

The variables of the above four functions will be denoted, respectively, as time, frequency, lag, and doppler. In time-frequency analysis [1]-[5], we extend these four functions of one variable to quadratic functions of two variables. Some examples of these quadratic functions are the the local auto-correlation function (LACF) of the signal, the LACF of the spectrum, the Wigner distribution, and the ambiguity function. These four functions are defined, respectively, as

$$
\begin{aligned}
R_{x}(t, \tau) & =x\left(t+\frac{\tau}{2}\right) x^{*}\left(t-\frac{\tau}{2}\right) \\
R_{X}(\theta, \omega) & =X\left(\omega+\frac{\theta}{2}\right) X^{*}\left(\omega-\frac{\theta}{2}\right) \\
& =\iint R_{x}(t, \tau) e^{-j(\theta t+\omega \tau)} d t d \tau
\end{aligned}
$$

Manuscript received February 1, 1997; revised June 12, 1998. The associate editor coordinating the review of this paper and approving it for publication was Dr. Henrique Malvar.

J. C. O'Neill is with Boston University, Boston, MA 02215 USA (e-mail: jeffo@bu.edu).

W. J. Williams is with the Electrical Engineerring and Computer Science Department, University of Michigan, Ann Arbor, MI 48109 USA (e-mail: wjw@eecs.umich.edu).

Publisher Item Identifier S 1053-587X(99)01342-2.

$$
\begin{aligned}
W_{x}(t, \omega) & =\int R_{x}(t, \tau) e^{-j \omega \tau} d \tau \\
A_{x}(\theta, \tau) & =\int R_{x}(t, \tau) e^{-j t \theta} d t
\end{aligned}
$$

and are all related to each other via Fourier transforms.

However, there are many other quadratic functions of the signal similar to the four defined above. For example, there are many "meaningful" functions of time and frequency. Some examples are spectrograms, the Rihaczek distribution, and the exponential distribution by Choi and Williams. As a means for encompassing the different representations of a signal, we will loosely define the concept of a domain.

Definition: Given a set of variables $V$, a domain is the set of meaningful representations of the signal that are functions of the variables $V$.

The name assigned to a domain will correspond to the variables of the domain. For example, the signal is a representation of the signal in the time domain, and the spectrum is a representation of the signal in the frequency domain. Other meaningful representations of the signal in the time and frequency domains are the squared magnitude of the signal $|x(t)|^{2}$ and the squared magnitude of the spectrum $|X(\omega)|^{2}$. The functions listed above in (2) are members of the time-lag, doppler-frequency, time-frequency, and doppler-lag domains, respectively.

In (2), we list quadratic functions of two of the following four variables:

- time;

- frequency;

- lag;

- doppler.

In this paper, we provide a method for creating functions of three of the above four variables, as well as a function of all four variables that will be called the local ambiguity function. These functions will all be quadralinear or quartic functions of the signal. Although other quartic functions of have been considered in the context of time-frequency analysis [6]-[10], these functions depend solely on time and frequency variables. The functions defined here are fundamentally different in that they include variables of lag and doppler in addition to variables of time and frequency. Jones and Baraniuk [11] have considered a function of time, lag, and doppler, and we investigate in detail the relationships between their function and the functions defined here in Section VI.

The paper is organized as follows. In Section II, we define several one-dimensional (1-D) Wigner mappings and apply them to create four signal representations that are functions of three variables. In Section III, we define several twodimensional (2-D) Wigner mappings and apply them to create 
a function of all four variables. In Sections IV and V, we investigate the marginals of these newly defined functions and compute some examples that reveal properties of these functions. In Section VI, the specific functions are generalized to create classes of functions that are analogous to the Cohen class. In Section VII, we apply the new functions to developing signal-adaptive kernels for the Cohen class that are functions of time and frequency.

\section{WIGNER MAPPINGS}

In this section, we are going to create some operators (not necessarily linear) that are equivalent to the Fourier transform operator and the operation of computing a Wigner distribution from a signal [1]-[5]. The operators, which we will define, provide a level of abstraction that will simplify notation and provide a clearer explanation of the concepts. To simplify the language, we will refer to the signal and the spectrum as the "two linear functions" and the Wigner distribution, the ambiguity function, the temporal LACF, and the spectral LACF as "the four quadratic functions," even though there are clearly other linear and quadratic functions of the signal. The linear and quadratic functions and the relationships between then are well established [1]-[5].

The first operator will be called a time Fourier mapping (FM) and is equivalent to the well-known Fourier transform operator. The time FM will be denoted as ${ }^{1}$

$$
X(\omega)=\underset{t \rightarrow \omega}{\mathbf{F}^{-}}(x(t)) .
$$

Similarly, the frequency FM will be equivalent to the inverse Fourier transform operator and will be denoted as

$$
x(t)=\underset{\omega \rightarrow t}{\mathbf{F}^{+}}(X(\omega)) .
$$

We have purposely avoided using the word "inverse" in defining these mappings, even though one is clearly the inverse of the other. The reason for this will soon be clear. The time FM and the frequency FM provide a slightly more abstract approach to computing the spectrum from the signal and vice versa.

Next, we would like to create operators to compute the Wigner distribution from the signal and the spectrum. The time Wigner mapping (WM) will be defined as the operator that computes the Wigner distribution from the signal and will be denoted as

$$
W_{x}(t, \omega)=\underset{t \rightarrow(t, \omega)}{\mathbf{W}^{-}}(x(t)) .
$$

Similarly, the frequency WM will be defined as the operator that computes the Wigner distribution from the spectrum and will be denoted as

$$
W_{x}(t, \omega)=\underset{\omega \rightarrow(\omega, t)}{\mathbf{W}^{+}}(X(\omega)) .
$$

The above two equations indicate that the time WM of the signal is identical to the frequency WM of the spectrum. This is a well-known result [1]-[5] that we express in the notation of this section and will be applied later in this section.

\footnotetext{
${ }^{1}$ The "-" denotes the sign of the complex exponential in the Fourier transform.
}

Theorem 1: If $X(\omega)=\mathbf{F}_{t \rightarrow \omega}^{-}(x(t))$, then $\mathbf{W}_{t \rightarrow(t, \omega)}^{-}(x(t))$ $=\mathbf{W}_{\omega \rightarrow(\omega, t)}^{+}(X(\omega))$.

By applying the Fourier mappings and Wigner mappings, we can convert between the two linear functions, between the four quadratic functions, and from the two linear functions to the four quadratic functions. Since the Wigner mappings are not inverses of each other, we chose to call them a time Wigner mapping and a frequency Wigner mapping. The Fourier mappings are inverses of each other, but, to be consistent, we chose to call them a time Fourier mapping and a frequency Fourier mapping (as opposed to a Fourier mapping and an inverse Fourier mapping).

Each of the four quadratic functions depend on two of the four variables of time, frequency, lag, and doppler. The creation of the Wigner mappings provides a method for creating functions that depend on three of the four variables and represent the signal in new domains. To do this, compare the operation of the time FM with the time WM

$$
\begin{aligned}
X(\omega) & =\underset{t \rightarrow \omega}{\mathbf{F}^{-}}(x(t)) \\
W_{x}(t, \omega) & =\underset{t \rightarrow(t, \omega)}{\mathbf{W}^{-}}(x(t)) .
\end{aligned}
$$

The time FM converts the signal from the (linear) signal to the (linear) spectrum, whereas the time WM converts the (linear) signal to the (quadratic) Wigner distribution. To obtain a joint representation, we simply replace a Fourier mapping with a Wigner mapping. This can be used to create quadralinear or quartic functions of three variables. For example, the temporal LACF and the ambiguity function are related through a time FM

$$
A_{x}(\theta, \tau)=\underset{t \rightarrow \theta}{\mathbf{F}^{-}}\left(R_{x}(t, \tau)\right) .
$$

To obtain a function of time, lag, and doppler, we simply replace the time FM in (3) with a time WM

$$
Q_{x}^{\omega}(t, \tau, \theta)=\underset{t \rightarrow(t, \theta)}{\mathbf{W}^{-}}\left(R_{x}(t, \tau)\right)
$$

where the choice of notation will become clear in a subsequent section. This function will be unimaginatively called the time-lag-doppler function (TLDF) and is a member of the time-lag-doppler domain. From Theorem 1, we know that we can also compute the TLDF by applying a frequency WM to the ambiguity function

$$
Q_{x}^{\omega}(t, \tau, \theta)=\underset{\theta \rightarrow(\theta, t)}{\mathbf{W}_{x}^{+}}\left(A_{x}(\theta, \tau)\right)
$$

By applying the above method to the other quadratic functions, we can create three other quartic functions that are members of a corresponding three-variable domain. These new functions will be called the frequency-lag-doppler function (FLDF), the time-frequency-lag function (TFLF), and the time-frequency-doppler function (TFDF). They are defined, respectively, as

$$
\begin{aligned}
Q_{x}^{t}(\omega, \tau, \theta) & =\underset{\tau \rightarrow(\tau, \omega)}{\mathbf{W}^{-}}\left(A_{x}(\theta, \tau)\right)=\underset{\omega \rightarrow(\omega, \tau)}{\mathbf{W}^{+}}\left(R_{X}(\theta, \omega)\right) \\
Q_{x}^{\theta}(t, \omega, \tau) & =\underset{\tau \rightarrow(\tau, \omega)}{\mathbf{W}^{-}}\left(R_{x}(t, \tau)\right)=\underset{\omega \rightarrow(\omega, \tau)}{\mathbf{W}^{+}}\left(W_{x}(t, \omega)\right) \\
Q_{x}^{\tau}(t, \omega, \theta) & =\underset{t \rightarrow(t, \theta)}{\mathbf{W}^{-}}\left(W_{x}(t, \omega)\right)=\underset{\theta \rightarrow(\theta, t)}{\mathbf{W}^{+}}\left(R_{X}(\theta, \omega)\right) .
\end{aligned}
$$


It is easily seen that the four, newly defined, quartic functions will always be real valued. Unlike the four quadratic functions, we can not convert a signal between the four quartic functions. We will now investigate each of the quartic functions in more detail.

\section{A. The Time-Lag-Doppler Function}

The TLDF can be written simply in terms of the signal

$$
\begin{aligned}
Q_{x}^{\omega}(t, \tau, \theta)= & \int x\left(t+\frac{\eta}{2}+\frac{\tau}{2}\right) x^{*}\left(t+\frac{\eta}{2}-\frac{\tau}{2}\right) \\
& \times x^{*}\left(t-\frac{\eta}{2}+\frac{\tau}{2}\right) x\left(t-\frac{\eta}{2}-\frac{\tau}{2}\right) e^{-j \eta \theta} d \eta .
\end{aligned}
$$

As seen from (7), the TLDF is clearly a quartic function of the signal. We will now show two interesting properties of this function. First, the TLDF of $x(t)$ and $x(t) e^{j\left(a_{0}+a_{1} t\right)}$ are the same; therefore, the TLDF is invariant to phase and frequency shifts. However, the TLDF will be covariant to time shifts. Second, the TLDF can be interpreted as a time-localized ambiguity function. ${ }^{2}$ We will demonstrate this second property analytically for a simple signal and computationally for a more complicated signal.

For a signal with a quadratic instantaneous frequency (IF)

$$
x(t)=e^{j\left(a_{0}+a_{1} t+a_{2} t^{2}+a_{3} t^{3}\right)}
$$

the TLDF has a simple, closed-form solution ${ }^{3}$

$$
Q_{x}^{\omega}(t, \tau, \theta)=\delta\left(\theta-\tau\left(2 a_{2}+6 a_{3} t\right)\right)
$$

We will show that the TLDF of this signal can be interpreted as a time-localized ambiguity function. To do this, we will locally approximate the signal $x(t)$ with chirp functions. At time $t_{0}$, the chirp $y(t)$ that provides the "best" approximation to $x(t)$ is

$$
y(t)=e^{j\left(c+\left(a_{1}-3 a_{3} t_{0}^{2}\right) t+\left(a_{2}+3 a_{3} t_{0}\right) t^{2}\right)}
$$

where $c$ is an arbitrary constant. Our meaning of "best" is illustrated with an example in Fig. 1. At time $t_{0}$, the instantaneous frequency and the derivative of the instantaneous frequency of $x(t)$ and $y(t)$ will be the same. The magnitude of the ambiguity function of $y(t)$ is

$$
\left|A_{y}(\theta, \tau)\right|=\delta\left(\theta-\tau\left(2 a_{2}+6 a_{3} t_{0}\right)\right)
$$

which is identical to (8) evaluated at $t=t_{0}$. This will clearly hold for any time $t_{0}$. Thus, for the quadratic chirp, the TLDF can be interpreted as a time-localized ambiguity function.

The interpretation of the TLDF as a time-localized ambiguity function also holds for more complicated signals. For example, consider a signal with a sinusoidal instantaneous frequency, whose Wigner distribution and ambiguity function

\footnotetext{
${ }^{2}$ We could also think of the TLDF as a doppler localized temporal LACF function, but this is not as intuitive.

${ }^{3}$ Note that the Wigner distribution of this signal does not have a simple, closed-form expression.
}

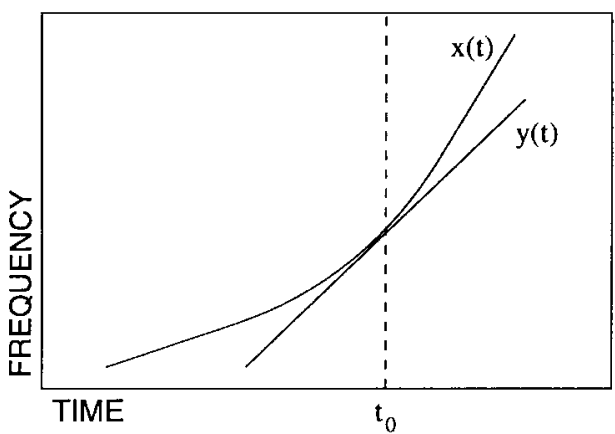

Fig. 1. Approximation of a quadratic chirp with a linear chirp.

are shown in Fig. 2. Fig. 2 also contains examples of the TLDF of this signal at time 48 and at time 64 . We can see that the TLDF of the signal at time 48 and time 64 is similar to the ambiguity functions of the chirps that locally approximate the signal at time 48 and at time 64 . The ambiguity function of the signal is dominated by the characteristics of the signal near time 64 since this is where the signal contains most of its energy. The TLDF appears to give meaningful information about the ambiguity function that is localized in time.

\section{B. The Frequency-Lag-Doppler Function}

The FLDF can be written simply in terms of the spectrum of the signal

$$
\begin{aligned}
Q_{x}^{t}(\omega, \tau, \theta)= & \frac{1}{2 \pi} \int X\left(\omega+\frac{\eta}{2}+\frac{\theta}{2}\right) X^{*}\left(\omega+\frac{\eta}{2}-\frac{\theta}{2}\right) \\
& \times X^{*}\left(\omega-\frac{\eta}{2}+\frac{\theta}{2}\right) X\left(\omega-\frac{\eta}{2}-\frac{\theta}{2}\right) e^{j \eta \tau} d \eta
\end{aligned}
$$

Comparing the above with the TLDF in (7), it is clear that FLDF can be considered to be the dual of the TLDF. The properties of the FLDF will also be dual to the properties of the TLDF.

- The FLDF will be invariant to phase shifts and time shifts but covariant to frequency shifts.

- The FLDF of a signal with a quadratic group delay (GD) will have a simple form analogous to (8).

- The FLDF can be interpreted as a frequency localized ambiguity function.

\section{The Time-Frequency-Lag Function}

The TFLF can be written simply in terms of the signal

$$
\begin{aligned}
Q_{x}^{\theta}(t, \omega, \tau)= & \int x\left(t+\frac{\tau}{2}+\frac{\gamma}{4}\right) x^{*}\left(t-\frac{\tau}{2}-\frac{\gamma}{4}\right) \\
& \times x^{*}\left(t+\frac{\tau}{2}-\frac{\gamma}{4}\right) x\left(t-\frac{\tau}{2}+\frac{\gamma}{4}\right) e^{-j \gamma \omega} d \gamma
\end{aligned}
$$

This function looks similar to the previous two, but its properties are quite different. 


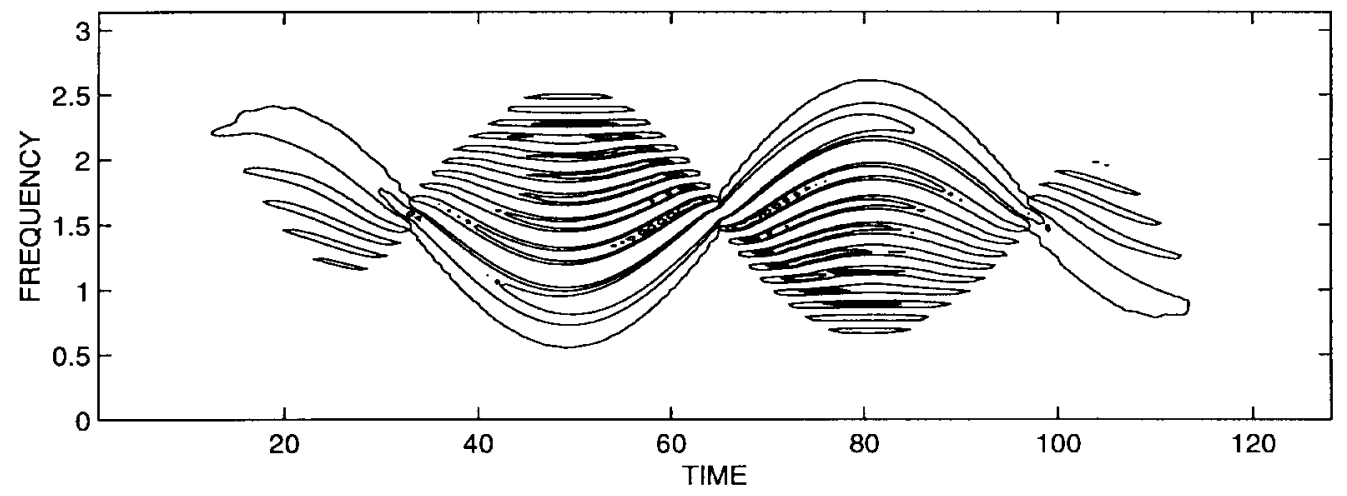

(a)

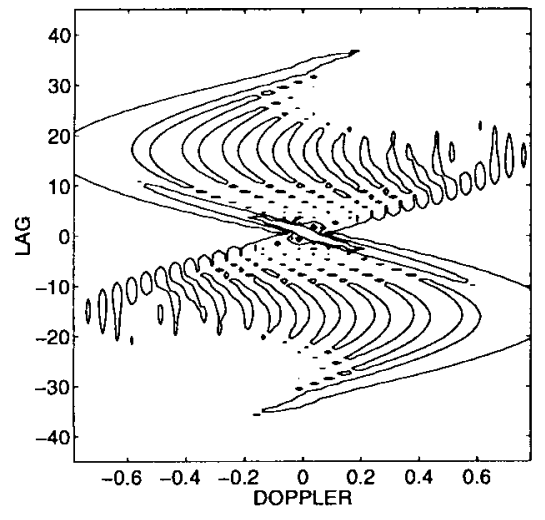

(b)

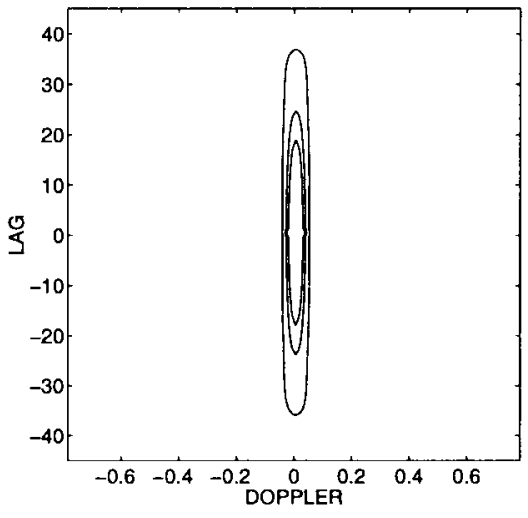

(c)

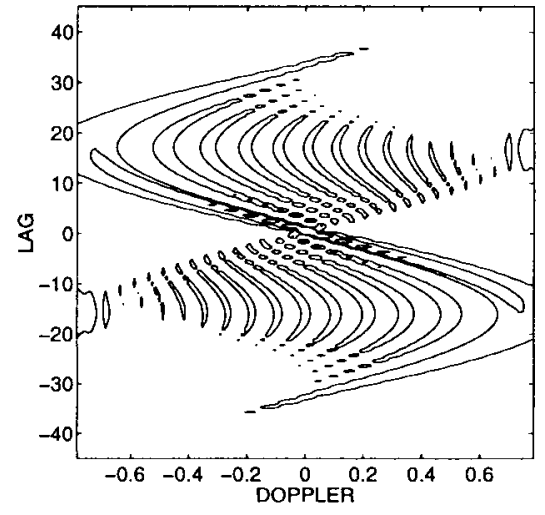

(d)

Fig. 2. Wigner distribution, ambiguity function, and TLDF of a signal with a sinusoidal frequency modulation (a) Wigner distribution. (b) Ambiguity function. (c) TLDF at time 48. (d) TLDF at time 64.

- The TFLF will be covariant to both time shifts and frequency shifts.

- There is no simple expression for the TFLF of a signal with a quadratic IF or a quadratic GD.

- The TFLF of a chirp $x(t)=e^{j\left(a_{0}+a_{1} t+a_{2} t^{2}\right)}$ is identical to the Wigner distribution of the chirp.

\section{The Time-Frequency-Doppler Function}

The TFDF can be written simply in terms of the spectrum of the signal

$$
\begin{aligned}
Q_{x}^{\tau}(t, \omega, \theta)= & \frac{1}{2 \pi} \int X\left(\omega+\frac{\theta}{2}+\frac{\gamma}{4}\right) X^{*}\left(\omega-\frac{\theta}{2}-\frac{\gamma}{4}\right) \\
& \times X^{*}\left(\omega+\frac{\theta}{2}-\frac{\gamma}{4}\right) X\left(\omega-\frac{\theta}{2}+\frac{\gamma}{4}\right) e^{j \gamma t} d \gamma
\end{aligned}
$$

Comparing the above with (10), we can see that the TFDF is the dual of the TFLF. In addition, the properties of the TFLF also hold for the TFDF.

\section{TWO-DIMENSIONAL WIGNER MAPPINGS}

Next, we would like to create a function that depends on all four of the variables of interest. To do this, we will focus on the relationship between the temporal LACF and the spectral
LACF

$$
R_{X}(\theta, \omega)=\underset{\tau \rightarrow \omega}{\mathbf{F}^{-}}\left(\underset{t \rightarrow \theta}{\mathbf{F}^{-}}\left(R_{x}(t, \tau)\right)\right)
$$

As before, we can replace the time FM's with time WM's to obtain a function of all four variables. However, since the time WM operator does not commute

$$
\underset{\tau \rightarrow(\tau, \omega)}{\mathbf{W}^{-}}\left(\underset{t \rightarrow(t, \theta)}{\mathbf{W}^{-}}\left(R_{x}(t, \tau)\right)\right) \neq \underset{t \rightarrow(t, \theta)}{\mathbf{W}^{-}}\left(\underset{\tau \rightarrow(\tau, \omega)}{\mathbf{W}^{-}}\left(R_{x}(t, \tau)\right)\right)
$$

and we will have two different functions depending on the order of the operators. In addition, this function would be an eighth-order function of the signal rather than a fourth-order function of the signal. This is undesirable since we would like the different methods for defining this function to be consistent. It would also be preferable for this function to be a quartic function of the signal.

We will approach this problem from a slightly different perspective by creating a 2-D time FM operator that will be identical to applying two 1-D time FM's in succession. The 2-D time FM will be denoted as

$$
R_{X}(\theta, \omega)=\underset{\substack{t \rightarrow \theta \\ \tau \rightarrow \omega}}{\mathbf{F}^{-,-}}\left(R_{x}(t, \tau)\right) .
$$

Similarly, we will also define a 2-D frequency FM denoted as

$$
R_{x}(t, \tau)=\underset{\substack{\theta \rightarrow t \\ \omega \rightarrow \tau}}{\mathbf{F}^{+,+}}\left(R_{X}(\theta, \omega)\right)
$$




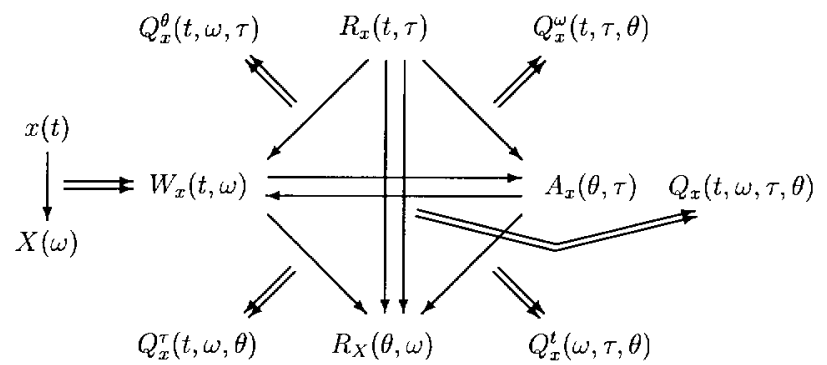

Fig. 3. Relationships between the linear, quadratic, and quartic functions. Single arrows represent Fourier mappings, and double arrows represent Wigner mappings.

and a 2-D mixed FM denoted as

$$
W_{x}(t, \omega)=\underset{\substack{\tau \rightarrow \omega \\ \theta \rightarrow t}}{\mathbf{F}^{-,+}}\left(A_{x}(\theta, \tau)\right)
$$

Next, we will create 2-D Wigner mappings that will correspond to the above 2-D Fourier mappings. First, a 2-D time WM will be identical to the operation that computes a 2-D Wigner distribution from an image

$$
W_{z}\left(t_{1}, t_{2}, \omega_{1}, \omega_{2}\right)=\underset{\substack{t_{2} \rightarrow\left(t_{2}, \omega_{2}\right) \\ t_{1} \rightarrow\left(t_{1}, \omega_{1}\right)}}{\mathbf{W}^{-,-}}\left(z\left(t_{1}, t_{2}\right)\right) .
$$

The 2-D frequency WM mapping will be identical to the operation that computes a 2-D Wigner distribution from the spectrum of the image

$$
W_{z}\left(t_{1}, t_{2}, \omega_{1}, \omega_{2}\right)=\underset{\substack{\omega_{2} \rightarrow\left(\omega_{2}, t_{2}\right) \\ \omega_{1} \rightarrow\left(\omega_{1}, t_{1}\right)}}{\mathbf{W}^{+,+}}\left(Z\left(\omega_{1}, \omega_{2}\right)\right) .
$$

Finally, the 2-D mixed WM will be defined and denoted as expected.

These 2-D mappings provide a more desirable method for computing a function of all four variables. To define this function, we will again use the relationship between the temporal LACF and the spectral LACF

$$
R_{X}(\theta, \omega)=\underset{\substack{\tau \rightarrow \omega \\ t \rightarrow \theta}}{\mathbf{F}^{-,-}}\left(R_{x}(t, \tau)\right)
$$

To define what we will call the local ambiguity function (LAF), we will replace the 2-D time FM with a 2-D time WM

$$
Q_{x}(t, \omega, \tau, \theta)=\underset{\substack{\tau \rightarrow(\tau, \omega) \\ t \rightarrow(t, \theta)}}{\mathbf{W}^{-,-}}\left(R_{x}(t, \tau)\right) .
$$

It can be shown that the methods for deriving the LAF are consistent within this framework.

Theorem 2:

$$
\begin{aligned}
\underset{\tau \rightarrow(\tau, \omega)}{\mathbf{W}_{t \rightarrow(t, \theta)}^{-,-}}\left(R_{x}(t, \tau)\right)= & \underset{\substack{\omega \rightarrow(\omega, \tau) \\
\theta \rightarrow(\theta, t)}}{\mathbf{W}^{+,+}}\left(R_{X}(\theta, \omega)\right) \\
= & \underset{\substack{t \rightarrow(t, \theta) \\
\omega \rightarrow(\omega, \tau)}}{\mathbf{W}^{-,+}}\left(W_{x}(t, \omega)\right) \\
= & \underset{\substack{\tau \rightarrow(\tau, \omega) \\
\theta \rightarrow(\theta, t)}}{\mathbf{W}^{-}++}\left(A_{x}(\theta, \tau)\right) .
\end{aligned}
$$

The proof of this theorem is straightforward, so it will not be presented here. A summary of the relationships between the two linear functions, the four quadratic functions, and the five quartic functions is shown in Fig. 3.

\section{MARgInALS}

Given a distribution function, a marginal is obtained by integrating over one of the variables of the function. The marginals of the Wigner distribution are

$$
\begin{aligned}
|x(t)|^{2} & =\int W_{x}(t, \omega) d \omega \\
|X(\omega)|^{2} & =\int W_{x}(t, \omega) d t .
\end{aligned}
$$

Marginals can be computed from the quartic functions analogous to the marginals of the quadratic functions. We will call the integral of the quartic functions over one variable a first-order marginal, over two variables a second-order marginal, and so forth. Fortuitously, the first-order marginals of the LAF are the other four quartic functions

$$
\begin{aligned}
& \int Q_{x}(t, \omega, \tau, \theta) d \omega=Q_{x}^{\omega}(t, \tau, \theta) \\
& \int Q_{x}(t, \omega, \tau, \theta) d \theta=Q_{x}^{\theta}(t, \omega, \tau) \\
& \int Q_{x}(t, \omega, \tau, \theta) d \tau=Q_{x}^{\tau}(t, \omega, \theta) \\
& \int Q_{x}(t, \omega, \tau, \theta) d t=Q_{x}^{t}(\omega, \tau, \theta) .
\end{aligned}
$$

This property motivated the choice of notation for the quartic functions.

The second-order marginals of the LAF will be the firstorder marginals of the other four quartic functions. Because of this, we will now only consider the marginals of the LAF. The second-order marginals provide some more fortuitous results as in

$$
\begin{aligned}
& \iint Q_{x}(t, \omega, \tau, \theta) d \omega d \theta=\left|R_{x}(t, \tau)\right|^{2} \\
& \iint Q_{x}(t, \omega, \tau, \theta) d t d \tau=\left|R_{X}(\theta, \omega)\right|^{2} \\
& \iint Q_{x}(t, \omega, \tau, \theta) d \tau d \theta=\left|W_{x}(t, \omega)\right|^{2} \\
& \iint Q_{x}(t, \omega, \tau, \theta) d t d \omega=\left|A_{x}(\theta, \tau)\right|^{2} .
\end{aligned}
$$

There are two other second-order marginals that would be members of time-doppler and lag-frequency domains. However, since quadratic functions of these variables do not exist, their meaning is unclear.

The third-order marginals also provide interesting relationships as in

$$
\begin{aligned}
\iiint Q_{x}(t, \omega, \tau, \theta) d \tau d \omega d \theta & =2|x(2 t)|^{2} *|x(2 t)|^{2}=\tilde{x}(t) \\
\iiint Q_{x}(t, \omega, \tau, \theta) d t d \omega d \theta & =r_{|x|^{2}}(\tau) \\
\iiint Q_{x}(t, \omega, \tau, \theta) d \tau d t d \theta & =2|X(2 \omega)|^{2} *|X(2 \omega)|^{2} \\
& =\tilde{X}(\omega) \\
\iiint Q_{x}(t, \omega, \tau, \theta) d \tau d t d \omega & =r_{|X|^{2}}(\theta)
\end{aligned}
$$




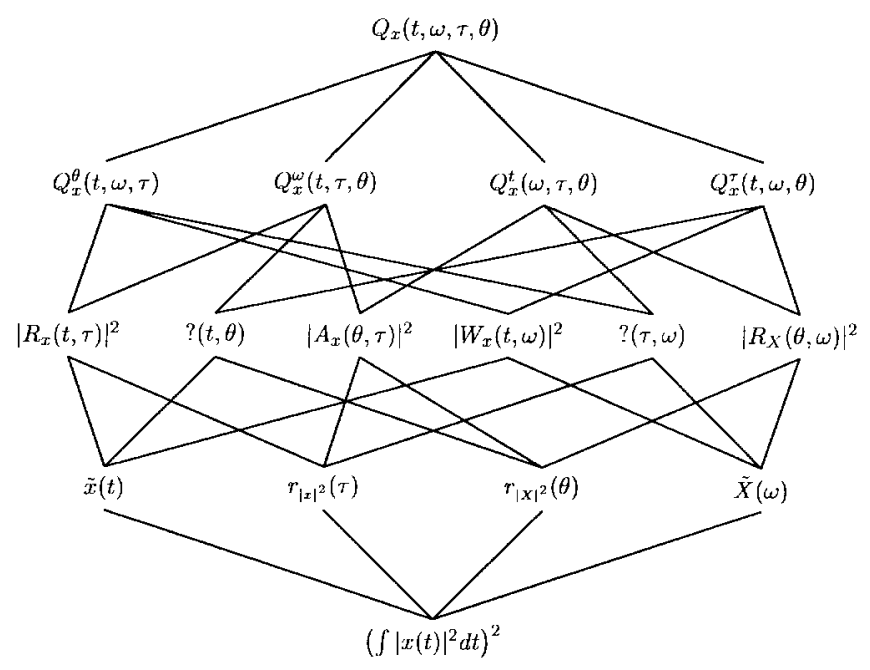

Fig. 4. Marginals of the LAF. The lines represent integration with respect to one of the variables of the function.

where

* convolution;

$r_{|x|^{2}}(\tau)$ auto-correlation function of $|x(t)|^{2}$;

$r_{|X|^{2}}(\theta)$ auto-correlation function of $|X(\omega)|^{2}$.

Finally, the fourth-order marginal of the LAF is

$$
\iiint \int Q_{x}(t, \omega, \tau, \theta) d t d \omega d \tau d \theta=\left(\int|x(t)|^{2} d t\right)^{2}
$$

The marginals of the quartic functions are summarized in Fig. 4.

\section{Properties of the Local Ambiguity Function}

We will now investigate the LAF in more detail. The LAF can be expressed simply in terms of both the signal and the spectrum as in

$$
\begin{aligned}
& Q_{x}(t, \omega, \tau, \theta) \\
& =\iint x\left(t+\frac{\eta}{2}+\frac{\tau}{2}+\frac{\gamma}{4}\right) \\
& \quad \times x^{*}\left(t+\frac{\eta}{2}-\frac{\tau}{2}-\frac{\gamma}{4}\right) x^{*}\left(t-\frac{\eta}{2}+\frac{\tau}{2}-\frac{\gamma}{4}\right) \\
& \quad \times x\left(t-\frac{\eta}{2}-\frac{\tau}{2}+\frac{\gamma}{4}\right) e^{-j \eta \theta} e^{-j \gamma \omega} d \eta d \gamma \\
& Q_{x}(t, \omega, \tau, \theta) \\
& =\left(\frac{1}{2 \pi}\right)^{2} \iint X\left(\omega+\frac{\eta}{2}+\frac{\theta}{2}+\frac{\gamma}{4}\right) \\
& \quad \times X^{*}\left(\omega+\frac{\eta}{2}-\frac{\theta}{2}-\frac{\gamma}{4}\right) X^{*}\left(\omega-\frac{\eta}{2}+\frac{\theta}{2}-\frac{\gamma}{4}\right) \\
& \quad \times X\left(\omega-\frac{\eta}{2}-\frac{\theta}{2}+\frac{\gamma}{4}\right) e^{j \gamma t} e^{j \eta \tau} d \eta d \gamma
\end{aligned}
$$

and, like the Wigner distribution, is self dual. To gain further insight into the LAF, we will analytically compute this function for some simple signals. For the first example, let our signal be a Gaussian windowed chirp

$$
x(t)=e^{j\left(a_{0}+a_{1} t+a_{2} t^{2}\right)} e^{-c t^{2}} .
$$

The LAF of this chirp is

$$
Q_{x}(t, \omega, \tau, \theta)=\left|W_{x}(t, \omega)\right|^{2}\left|A_{x}(\theta, \tau)\right|^{2} .
$$

The LAF has properties corresponding to an ambiguity function localized in time and frequency (similar to the TLDF and the FLDF). For $t$ and $\omega$ that correspond to the instantaneous frequency of the signal

$$
\omega \approx a_{1}+2 a_{2} t \text { and } t \approx 0
$$

the LAF will be a constant times the squared magnitude of the ambiguity function of the chirp. For $t$ and $\omega$ that do not satisfy the above conditions, the LAF will be essentially zero.

For a chirp function, the ambiguity function is, in a sense, "stationary." What we mean by this is that the "localized" ambiguity function is the same for all parts of the signal. This is revealed in the LAF since the time and frequency variables are separable from the lag and doppler variables. For more complicated functions, like the one shown in Fig. 2, the variables will not be separable since the ambiguity function will not be "stationary." Examples of these more complicated signals show that the LAF still satisfies an intuitive notion of being a time and frequency localized ambiguity function.

For a two-component signal, the situation becomes more complicated. Since the LAF is quartic rather than quadratic, there will be more cross terms for multicomponent signals than in quadratic functions like the Wigner distribution. In the general case, the LAF of a two component signal $x(t)=$ $x_{1}(t)+x_{2}(t)$ will be

$Q_{x}(t, \omega, \tau, \theta)=Q_{x_{1}}(t, \omega, \tau, \theta)+Q_{x_{2}}(t, \omega, \tau, \theta)+$ other terms.

We will use time- and frequency-shifted versions of a Gaussian signal

$$
x_{0}(t)=e^{-t^{2} / 2}
$$

to compare the cross terms in the Wigner distribution with the cross terms in the LAF. The Wigner distribution and LAF of the Gaussian signal $x_{0}(t)$ are multidimensional Gaussians

$$
\begin{aligned}
W_{x_{0}}(t, \omega) & =e^{-\left(t^{2}+\omega^{2}\right)} \\
Q_{x_{0}}(t, \omega, \tau, \theta) & =e^{-\left(2 t^{2}+2 \omega^{2}+\tau^{2} / 2+\theta^{2} / 2\right)} .
\end{aligned}
$$

We will now consider the two-component signal

$$
x(t)=x_{0}\left(t-t_{1}\right) e^{j \omega_{1} t}+x_{0}\left(t-t_{2}\right) e^{j \omega_{2} t}
$$

and define the following quantities to simplify notation:

$$
\begin{aligned}
\Delta_{t} & =t_{2}-t_{1} \\
\Delta_{\omega} & =\omega_{2}-\omega_{1} \\
\bar{t} & =\frac{t_{1}+t_{2}}{2} \\
\bar{\omega} & =\frac{\omega_{1}+\omega_{2}}{2} .
\end{aligned}
$$

The Wigner distribution of this signal is

$$
\begin{aligned}
W_{x}(t, \omega)= & W_{x_{0}}\left(t-t_{1}, \omega-\omega_{1}\right)+W_{x_{0}}\left(t-t_{2}, \omega-\omega_{2}\right) \\
& +2 W_{x_{0}}(t-\bar{t}, \omega-\bar{\omega}) \cos \left(t \Delta_{\omega}-\omega \Delta_{t}-\Delta_{t} \bar{\omega}\right)
\end{aligned}
$$




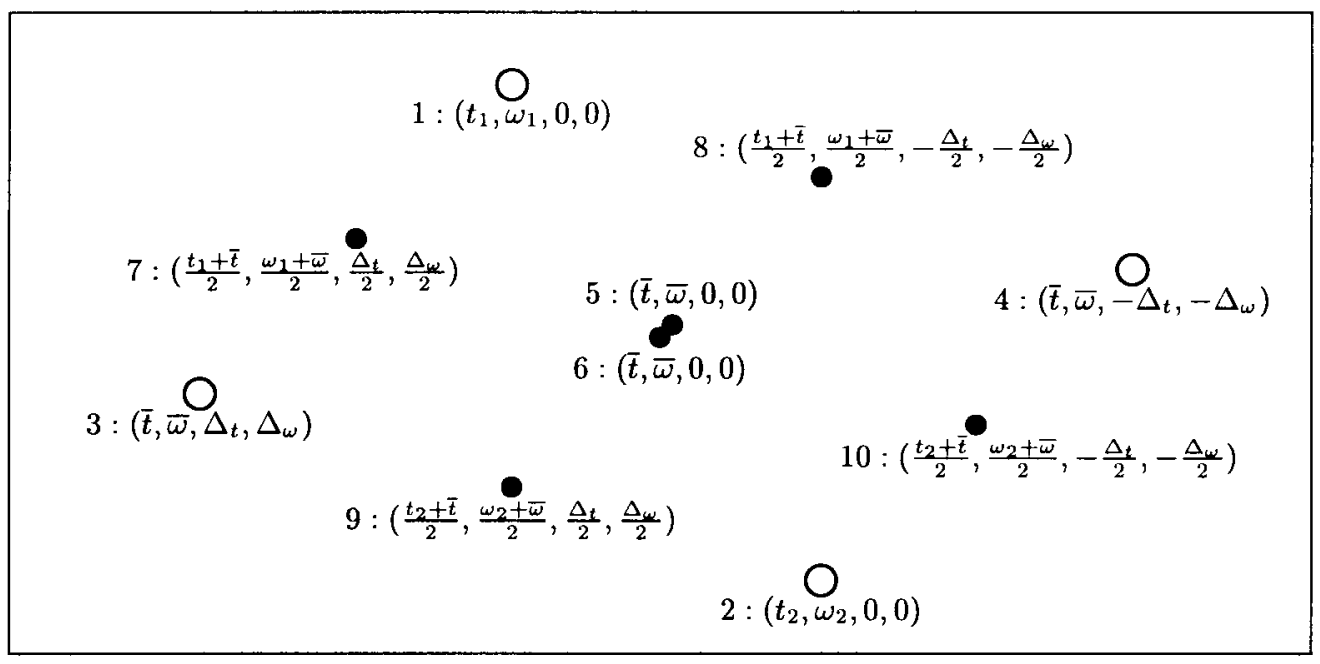

Fig. 5. Geometry of the LAF for a two component signal (four dimensions flattened into two dimensions). The open circles represent the auto terms, and the dark circles represent the cross terms. Next to each circle is the number of the term in (12), along with the location in the four-dimensional space.

and consists of two auto terms and one cross term. The LAF of this signal is

$$
\begin{aligned}
Q_{x}( & t, \omega, \tau, \theta) \\
= & Q_{x_{0}}\left(t-t_{1}, \omega-\omega_{1}, \tau, \theta\right)+Q_{x_{0}}\left(t-t_{2}, \omega-\omega_{2}, \tau, \theta\right) \\
& +Q_{x_{0}}\left(t-\bar{t}, \omega-\bar{\omega}, \tau-\Delta_{t}, \theta-\Delta_{\omega}\right) \\
& +Q_{x_{0}}\left(t-\bar{t}, \omega-\bar{\omega}, \tau+\Delta_{t}, \theta+\Delta_{\omega}\right) \\
& +2 Q_{x_{0}}(t-\bar{t}, \omega-\bar{\omega}, \tau, \theta) \cos \left(2 t \Delta_{\omega}-2 \omega \Delta_{t}-\Delta_{t} \bar{\omega}\right) \\
& +2 Q_{x_{0}}(t-\bar{t}, \omega-\bar{\omega}, \tau, \theta) \cos \left(\tau \Delta_{\omega}-\theta \Delta_{t}\right) \\
& +2 Q_{x_{0}}\left(t-\frac{t_{1}+\bar{t}}{2}, \omega-\frac{\omega_{1}+\bar{\omega}}{4}, \tau-\frac{\Delta_{t}}{2}, \theta-\frac{\Delta_{\omega}}{2}\right) \\
& \times \cos \left(\left(t+\frac{\tau}{2}\right) \Delta_{\omega}-\left(\omega+\frac{\theta}{2}\right) \Delta_{t}-\Delta_{t} \bar{\omega}\right) \\
& +2 Q_{x_{0}}\left(t-\frac{t_{1}+\bar{t}}{2}, \omega-\frac{\omega_{1}+\bar{\omega}}{2}, \tau+\frac{\Delta_{t}}{2}, \theta+\frac{\Delta_{\omega}}{2}\right) \\
& \times \cos \left(\left(t-\frac{\tau}{2}\right) \Delta_{\omega}-\left(\omega-\frac{\theta}{2}\right) \Delta_{t}-\Delta_{t} \bar{\omega}\right) \\
& +2 Q_{x_{0}}\left(t-\frac{t_{2}+\bar{t}}{2}, \omega-\frac{\omega_{2}+\bar{\omega}}{2}, \tau-\frac{\Delta_{t}}{2}, \theta-\frac{\Delta_{\omega}}{2}\right) \\
& \times \cos \left(\left(t-\frac{\tau}{2}\right) \Delta_{\omega}-\left(\omega-\frac{\theta}{2}\right) \Delta_{t}-\Delta_{t} \bar{\omega}\right) \\
& +2 Q_{x_{0}}\left(t-\frac{t_{2}+\bar{t}}{2}, \omega-\frac{\omega_{2}+\bar{\omega}}{2}, \tau+\frac{\Delta_{t}}{2}, \theta+\frac{\Delta_{\omega}}{2}\right) \\
& \times \cos \left(\left(t+\frac{\tau}{2}\right) \Delta_{\omega}-\left(\omega+\frac{\theta}{2}\right) \Delta_{t}-\Delta_{t} \bar{\omega}\right)
\end{aligned}
$$

where we will call the first four terms auto terms and the last six terms cross terms.

The first two terms in (12) correspond to the LAF's of the individual Gaussians. The third and fourth terms represent the cross-correlation of the two Gaussians and are analogous to the cross-correlation terms that appear in the auto-correlation function of the signal and the spectrum

$$
\begin{aligned}
r_{x}(\tau) & =e^{-\left(\tau-\Delta_{t}\right)^{2} / 4}+2 e^{-\tau^{2} / 4}+e^{-\left(\tau+\Delta_{t}\right)^{2} / 4} \\
r_{X}(\theta) & =e^{-\left(\theta-\Delta_{\omega}\right)^{2} / 4}+2 e^{-\theta^{2} / 4}+e^{-\left(\theta+\Delta_{\omega}\right)^{2} / 4} .
\end{aligned}
$$

The auto-correlation functions have terms centered at $\tau=$ $\pm \Delta_{t}$ and $\theta= \pm \Delta_{\omega}$; therefore, a function of time, frequency, lag, and doppler should also have terms centered at $\tau= \pm \Delta_{t}$ and $\theta= \pm \Delta_{\omega}$. The first four terms of the LAF represent the distribution of the two Gaussians simultaneously in time, frequency, lag, and doppler. For these reasons, the first four terms of the LAF will be called auto terms analogous to the auto terms of the Wigner distribution.

The last six terms in (12) have properties that are very similar to the properties of cross terms in the Wigner distribution [5]. Each of the last the last six terms is centered directly between two of the first four terms. In addition, each of the last six terms is oscillatory, and the direction of the oscillation is perpendicular to the line connecting the two auto terms. Finally, between every pair of auto terms lies one of the last six terms. Again, for these reasons, the last six terms will be called cross terms. A pictorial representation of these ten terms is shown in Fig. 5, where the number of the term is indicated along with its position in four-dimensional (4-D) space.

\section{Classes of Quartic Functions}

The quartic functions that we have defined are, as we will show, similar to the Wigner distribution. By applying a kernel to the Wigner distribution, we can create an infinite number of time-frequency distributions. In the same way, we can apply kernels to the quartic functions to create an infinite number of distributions of three and four variables.

Jones and Baraniuk have developed a method for creating a function of time, lag, and doppler, which they call a short-time ambiguity function (STAF) [11]. They developed this function for the purpose of creating signal-adaptive kernels that vary over time. We will investigate this idea more extensively in the next section, but for now, we would like to compare the STAF with the five quartic functions and provide a means for creating other functions of three and four variables.

To compute the STAF at time $t_{0}$, first apply a window $h(t)$ to "localize" the signal to time $t_{0}$

$$
\tilde{x}_{t_{0}}(t)=x(t) h\left(t-t_{0}\right)
$$


and then compute the ambiguity function of this windowed signal

$$
\operatorname{STAF}_{x}\left(t_{0}, \tau, \theta ; h\right)=A_{\tilde{x}_{t_{0}}}(\theta, \tau) .
$$

This function, like the short-time Fourier transform, will be complex valued. Therefore, in practice, Jones and Baraniuk used the squared magnitude of this function, which we shall call an "ambiguigram"

$$
\mathrm{AG}_{x}(t, \tau, \theta ; h)=\left|\operatorname{STAF}_{x}(t, \tau, \theta ; h)\right|^{2} .
$$

The ambiguigram is similar to the spectrogram and provides an intuitive method for creating an ambiguity function that is localized in time.

We will now compare the methods for computing the ambiguigram and the TLDF with the methods for computing the Wigner distribution and the spectrogram. In the notation that we have established, the spectrogram and Wigner distribution can be computed as

$$
\begin{aligned}
\mathrm{SG}_{x}\left(t_{0}, \omega ; h\right) & =\left|\underset{t \rightarrow \omega}{\mathbf{F}^{-}}\left(\tilde{x}_{t_{0}}(t)\right)\right|^{2} \\
W_{x}(t, \omega) & =\underset{t \rightarrow(t, \omega)}{\mathbf{W}^{-}}(x(t))
\end{aligned}
$$

and the ambiguigram and TLDF can be computed as

$$
\begin{aligned}
\mathrm{AG}_{x}\left(t_{0}, \tau, \theta ; h\right) & =\left|\mathbf{F}_{t \rightarrow \theta}^{-}\left(R_{\tilde{x}_{t_{0}}}(t, \tau)\right)\right|^{2} \\
Q_{x}^{\omega}(t, \tau, \theta) & =\underset{t \rightarrow(t, \theta)}{\mathbf{W}}\left(R_{x}(t, \tau)\right)
\end{aligned}
$$

which shows that the relationship between the ambiguigram and the TLDF is analogous to the relationship between the spectrogram and the Wigner distribution. This is enlightening in that the tradeoffs between the ambiguigram and the TLDF are immediately apparent because of the established tradeoffs between the spectrogram and the Wigner distribution. Further, it is straightforward to create a class of time-lagdoppler functions similar to the Cohen class of time-frequency distributions

$$
\begin{aligned}
\mathcal{Q}_{x}^{\omega}(t, \tau, \theta ; \mathcal{P})= & \iiint Q_{x}^{\omega}(t, \tau, \theta) \\
& \times \mathcal{P}\left(t-t^{\prime}, \tau-\tau^{\prime}, \theta-\theta^{\prime}\right) d t^{\prime} d \tau^{\prime} d \theta^{\prime}
\end{aligned}
$$

where $\mathcal{P}(t, \tau, \theta)$ is a 3-D kernel function. Ambiguigrams will be members of this class where the kernel will be a function of the window $h(t)$.

In a similar manner, we can construct classes from the other four quartic functions. We shall present only the class for the LAF as it encompasses all of the others

$$
\begin{aligned}
& \mathcal{Q}_{x}(t, \omega, \tau, \theta ; \mathcal{P}) \\
& \quad=\iiint \int Q_{x}(t, \omega, \tau, \theta) \\
& \quad \times \mathcal{P}\left(t-t^{\prime}, \omega-\omega^{\prime}, \tau-\tau^{\prime}, \theta-\theta^{\prime}\right) d t^{\prime} d \omega^{\prime} d \tau^{\prime} d \theta^{\prime}
\end{aligned}
$$

where $\mathcal{P}(t, \omega, \tau, \theta)$ is a 4-D kernel function that is analogous to the kernel in the Cohen class. The above class generalizes the ambiguigram of Jones and Baraniuk in two ways. First, a method is given for creating an infinite number of functions of time, lag, and doppler. Second, their three-variable function is extended to create a function of all four variables. Since the purpose of creating the ambiguigram was to create signaladaptive kernels that vary over time, we will now investigate the use of the LAF to create signal-adaptive kernels that vary over time and frequency.

\section{AdAPTIVE KeRnel Design}

Time-frequency distributions in the Cohen class can be computed by convolving the Wigner distribution with a kernel function

$$
C_{x}(t, \omega ; \psi)=\iint W_{x}\left(t^{\prime}, \omega^{\prime}\right) \psi\left(t-t^{\prime}, \omega-\omega^{\prime}\right) d t^{\prime} d \omega^{\prime} .
$$

The purpose of the kernel is to filter out cross terms and maintain the resolution of the auto terms. Since the structure of the cross terms and auto terms in the Wigner distribution varies greatly for different signals, kernels that work well for one signal will not necessarily work well for other signals. Because of this, Baraniuk and Jones [12], [13] have proposed methods for creating signal adaptive kernels. Their algorithms use the ambiguity function as a means for finding "good" signaladaptive kernels. It has been observed that in the ambiguity function, the auto terms lie near the origin, whereas the cross terms tend to lie away from the origin [14], and the methods of Baraniuk and Jones exploit this observation.

The adaptive kernel methods [12], [13] extend the functionality of the Cohen class. However, there are further improvements that can be made to this method. The purpose of a TFD is to analyze signals whose characteristics change over time. The above methods choose a kernel that is adapted over the entire signal. However, since the signal is nonstationary, it is reasonable to assume that a kernel that works well at one time will not necessarily work well at another time. Jones and Baraniuk recognized this and extended their algorithm to design signal-adaptive, time-varying kernels [11]. This method can be formulated in the time-frequency plane as

$$
C_{x}(t, \omega ; \psi)=\iint W_{x}\left(t^{\prime}, \omega^{\prime}\right) \psi\left(t, t^{\prime}, \omega-\omega^{\prime} ; x\right) d t^{\prime} d \omega^{\prime}
$$

where the time-varying kernel is created by applying the radially Gaussian kernel (RGK) algorithm [12] to the ambiguigram that was introduced in the previous section.

We will now apply the RGK algorithm of Jones and Baraniuk to a smoothed version of the LAF for the purpose of creating signal-adaptive kernels that vary over time and frequency. There are two potential advantages in using the LAF over the ambiguigram. First, the LAF should be able to provide better resolution in the same way that the Wigner distribution provides better resolution than the spectrogram. Second, the LAF provides greater flexibility by allowing the kernel to vary over time and frequency instead of just over time.

The implementation of this method is conceptually simple but extremely expensive computationally. We will implement this method using the framework of the type II Cohen class that was introduced in [15]-[17]. The steps for implementing 


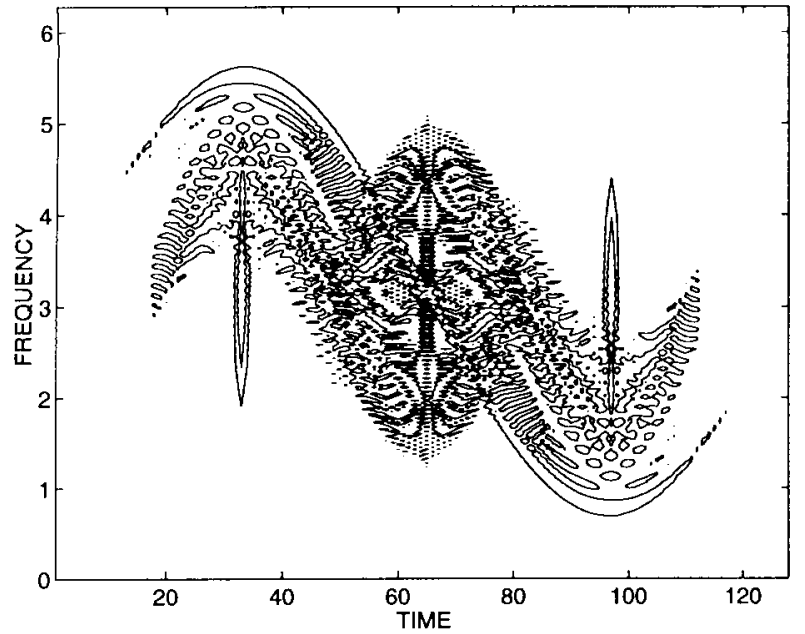

(a)

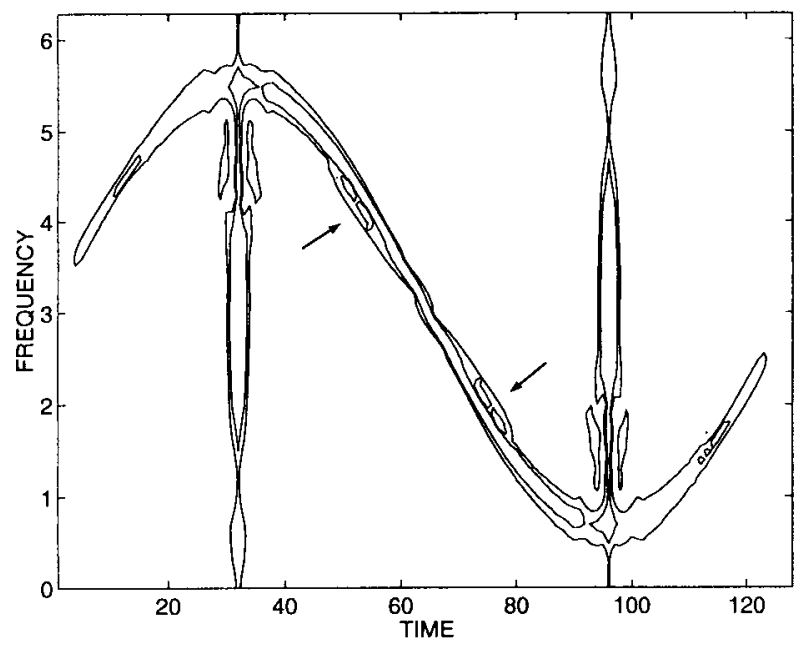

(c)

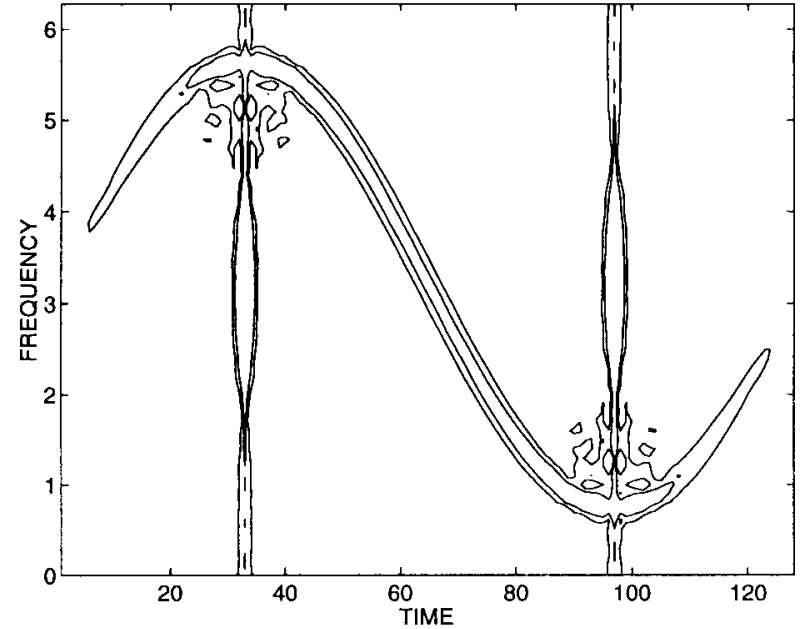

(b)

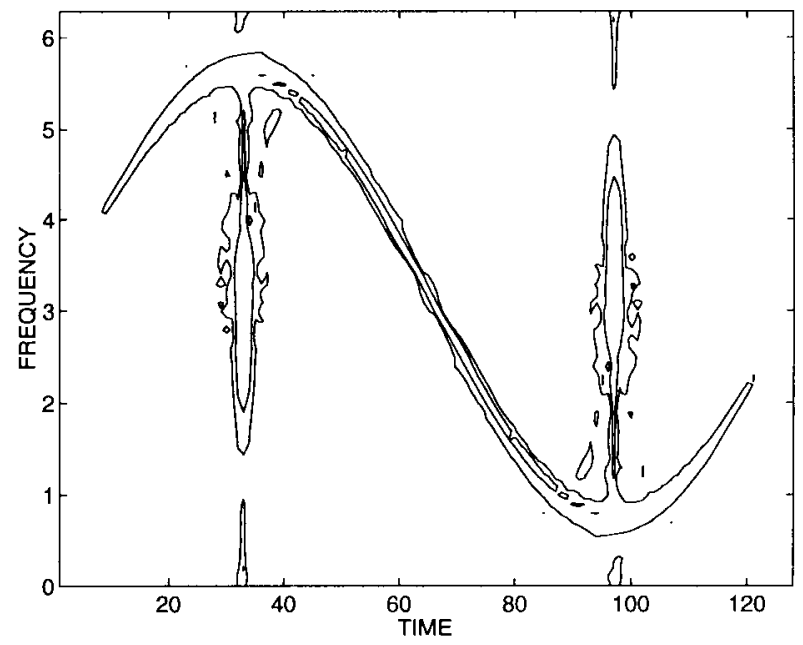

(d)

Fig. 6. Comparison of the Wigner distribution with the three different methods for the first example signal. (a) Wigner distribution. (b) Method 1. (c) Method 2. (d) Method 3.

the signal-adaptive, time-, and frequency-varying kernels are as follows.

- Compute the Wigner distribution $W_{x}(t, \omega)$ of the signal.

- Compute the LAF $Q_{x}(t, \omega, \tau, \theta)$ of the signal.

- Apply 4-D smoothing to $Q_{x}(t, \omega, \tau, \theta)$ to obtain $\mathcal{Q}_{x}(t, \omega, \tau, \theta)$. This is necessary since the cross terms in the LAF prevent the RGK algorithm from finding a "good" kernel. We used a 4-D version of [ $\left[\begin{array}{llll}0.25 & 0.5 & 0.25\end{array}\right]$ for our smoothing function.

- For each point $\left(t_{0}, \omega_{0}\right)$ in the time-frequency plane

-apply the RGK algorithm to $\mathcal{Q}_{x}\left(t_{0}, \omega_{0}, \tau, \theta\right)$ to compute the kernel at $\left(t_{0}, \omega_{0}\right)$;

- apply the kernel to calculate the TFD at $\left(t_{0}, \omega_{0}\right)$.

We will compare the TFD's generated from three different methods for kernel design.

Method 1) The first method uses the binomial kernel [18]. This kernel is fixed (does not vary over time nor frequency) and is not signal adaptive.

Method 2) The second method uses the ambiguigram to compute a signal-adaptive kernel that varies over time [11].
Method 3) The third method, as described above, uses the LAF to create a signal-adaptive kernel that varies over time and frequency.

All three methods have a window length parameter that provides a tradeoff between maintaining resolution of the auto terms and suppressing the cross terms. The window lengths used will be indicated in each example. Methods II and III also have "volume" parameter that is part of the RGK algorithm. In all examples, we will use a volume parameter of 4 .

It is difficult to compare different methods for computing TFD's as there is no clear measure of the "goodness" of a TFD. We will present pictures of the TFD's obtained by using the three different methods and provide a qualitative comparison of the cross term suppression and resolution. We will test the three different methods with two synthetic signals. In order to achieve as fair a comparison as possible, all TFD's have been normalized to have the same total volume, and the dynamic range of the images is the same within each example.

Example 1: The first test signal consists of three components: two short, Gaussian pulses centered at times 32 and 96 and a component with a sinusoidal frequency modulation. In 
Fig. 6, we present the Wigner distribution of this signal along with three TFD's computed by applying each of three methods detailed above. For this relatively simple signal, neither of the adaptive methods (methods II and III) provides a significant advantage over the much simpler binomial kernel (method I). There is a slight indication of the advantages of method III over method II in Figs. 6(c) and (d). Due to the poorer resolution of the ambiguigram, we can see cross terms close to the signal as indicated by the arrows in Fig. 6(c). We can also see that method III in Fig. 6(d) provides the highest resolution near time 64.

Example 2: The second test signal consists of four components: two complex exponentials at frequencies of 0 and $\pi \mathrm{rad}$ and two components with a sinusoidal instantaneous frequency (IF) centered at frequencies of $\pi / 2$ and $3 \pi / 2 \mathrm{rad}$. Time-frequency distributions are computed using the three different methods and are shown in Fig. 7. The binomial distribution represents the auto terms well but does not attenuate the cross terms as well as the other methods. Method II attenuates the cross terms better than the binomial distribution, but the sinusoidal IF components are "linearized." It is not clear what causes the linearization in the method II, but it could be that the RGK algorithm is adapting to the complex exponentials rather than the sinusoidal IF components. Method III provides the best cross term attenuation and does not suffer from the linearization seen in method II. Method III does not resolve the sinusoidal component well at time 80. The reason for this is that since the auto terms are close together at that point, the cross terms in the LAF are more difficult to attenuate, and this prevents the RGK algorithm method from finding a "good" kernel.

The methods of Jones and Baraniuk provide a clear means for increasing the versatility of the Cohen class. The LAF, which has been derived in this paper, provides a means for generalizing the ambiguigram of Jones and Baraniuk and further increasing the versatility of the Cohen class. In addition, the LAF provides insight into the ambiguigram in the same way that the Wigner distribution provides insight into the spectrogram. The kernels generated by the LAF provide some improvement over the kernels generated by the ambiguigram at the expense of greatly increased computations. Kernel design with the LAF may be too expensive computationally to be practical, but this example affirms that the LAF can indeed be applied as an ambiguity function localized in time and frequency.

\section{CONCLUSION}

In signal processing, we are often interested in the following four functions of one variable: the signal, the spectrum, autocorrelation function of the signal, and the auto-correlation function of the spectrum. In time-frequency analysis, we extend these functions of one variable to several quadratic functions of two variables. In this paper, we further extend the concepts of time-frequency analysis to create quartic functions of three and four of these variables. The function of all four variables has properties of an ambiguity function localized in time and frequency, and thus, it is called the local ambiguity

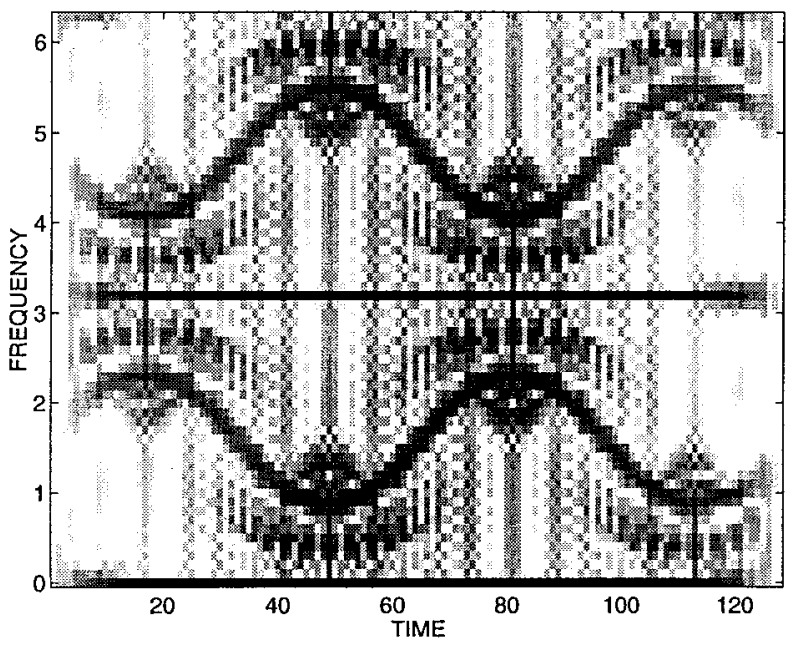

(a)

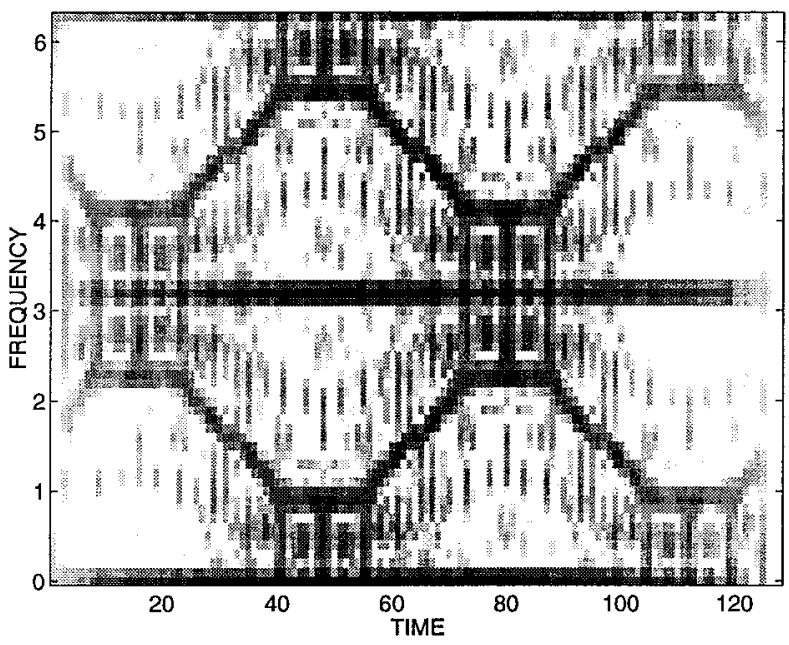

(b)

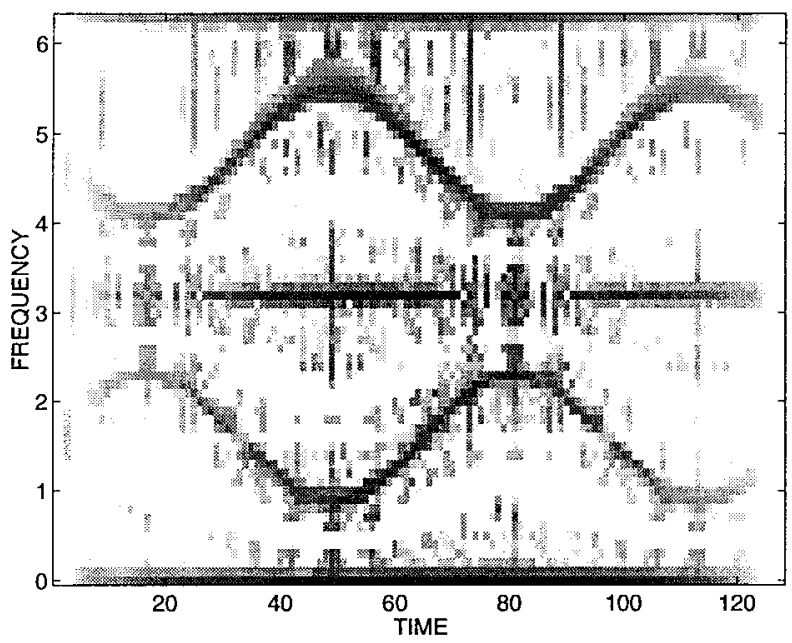

(c)

Fig. 7. Comparison of the three different methods for the second example signal. (a) Method 1. (b) Method 2. (c) Method 3.

function. The local ambiguity function is successfully applied to the design of time- and frequency-varying kernels for the Cohen class and shown to have advantages over previous methods at the cost of greatly increased computations. 


\section{REFERENCES}

[1] T. A. C. M. Claasen and W. F. G. Mecklenbräuker, "The Wigner distribution-A tool for time-frequency signal analysis, part I: Continuoustime signals," Philips J. Res., vol. 35, no. 3, pp. 217-250, 1980.

[2] _ "The Wigner distribution-A tool for time-frequency signal analysis, Part III: Relations with other time-frequency signal transformations," Philips J. Res., vol. 35, no. 6, pp. 372-389, 1980.

[3] L. Cohen, Time-Frequency Analysis. Englewood Cliffs, NJ: PrenticeHall, 1995.

[4] P. Flandrin, Temps-Fréquence. Paris, France: Hermès, 1993.

[5] F. Hlawatsch and G. F. Boudreaux-Bartels, "Linear and quadratic timefrequency signal representations," IEEE Signal Processing Mag., pp. 21-67, Apr. 1992.

[6] B. Boashash and P. O'Shea, " Polynomial Wigner-Ville distributions and their relationship to time-varying higher order spectra," IEEE Trans. Signal Processing, vol. 42, pp. 216-220, Jan. 1994.

[7] J. R. Fonollosa and C. L. Nikias, "Wigner higher order moment spectra: Definition, properties, computation, and application to transient signal analysis," IEEE Trans. Signal Processing, vol. 41, pp. 245-266, Jan. 1993.

[8] LJ. Stanković, "A multitime definition of the Wigner higher order distribution: L-Wigner distribution," IEEE Signal Processing Lett., vol. 1, pp. 106-109, July 1994.

[9] $\longrightarrow$, "A method for improved distribution concentration in the time-frequency analysis of multicomponent signals using the L-Wigner distribution," IEEE Trans. Signal Processing, vol. 43, pp. 1262-1268, May 1995.

[10] LJ. Stanković and S. Stankovic, "An analysis of instantaneous frequency representations using time-frequency distributions-Generalized Wigner distribution,” IEEE Trans. Signal Processing, vol. 43, pp. 549-552, Feb. 1995.

[11] D. L. Jones and R. G. Baraniuk, "An adaptive optimal kernel timefrequency representation," IEEE Trans. Signal Processing, vol. 43, pp. 2361-2371, Oct. 1995.
[12] R. G. Baraniuk and D. L. Jones, "Signal-dependent time-frequency analysis using a radially Gaussian kernel," Signal Process., vol. 32, pp. 263-284, June 1993.

[13] , "A signal-dependent time-frequency representation: Optimal kernel design," IEEE Trans. Signal Processing, vol. 41, pp. 1589-1602, Apr. 1993.

[14] P. Flandrin, "Some features of time-frequency representations of multicomponent signals," in Proc. IEEE Int. Conf. Acoust., Speech, Signal Process., 1984, pp. 41.B.4.1-41.B.4.4.

[15] J. C. O'Neill and W. J. Williams, "New properties for discrete, bilinear time-frequency distributions," in Proc. IEEE Int. Symp. Time-Freq. TimeScale Anal., 1996, pp. 509-512.

[16] _ _Shift covariant time-frequency distributions of discrete signals," IEEE Trans. Signal Processing, vol. 47, pp. 133-146, Jan. 1999.

[17] J. C. O'Neill, "Shift covariant time-frequency distributions of discrete signals," Ph.D. dissertation, Univ. Michigan, Ann Arbor, 1997. Available on-line at http://www. eecs.umich.edu/ jeffo.

[18] W. J. Williams and J. Jeong, "Reduced interference time-frequency distributions," in Time-Frequency Signal Analysis: Methods and Applications, B. Boashash, Ed. London, U.K.: Longman and Cheshire, 1991, ch. 3 .

[19] J. C. O'Neill and W. J. Williams, "Quadralinear time-frequency representations," in Proc. IEEE Int. Conf. Acoust., Speech, Signal Process. 1995, vol. 2, pp. 1005-1008.

Jeffrey C. O'Neill (M'98), for photograph and biography, see p. 146 of the January 1999 issue of this TRANSACTIONS.

William J. Williams (SM'73), for photograph and biography, see p. 146 of the January 1999 issue of this TRANSACTIONS. 